\title{
La epidemia de escarlatina del año 1929 en Chile
}

\author{
Enrique Laval $R$.
}

Pontificia Universidad Católica

de Chile, Santiago.

Facultad de Medicina.

Programa de Estudios Médicos

Humanísticos.

Recibido: 20 noviembre 2008 Aceptado: 27 noviembre 2008

Correspondencia a:

Enrique Laval Román revinf@sochinf.cl

\section{Scarlet fever epidemic during year 1929 in Chile}

Scarlet fever is endemic in Chile, with relatively low morbidity and periodic exacerbations every 4 or 5 years, which can become epidemics. From 1921 to 1927, the number of patients hospitalized in the country fluctuated from 15 to 65 per year, until an epidemic involving nearly 3.000 patients started at the end of 1928 and continued during all 1929. 978 patients died, 537 (52.5\%) were from Santiago. Public Health authorities confronted this emergency with prevention, prophylaxis, isolation and treatment measures and 558 beds were disposed for patient hospitalization. Vaccination trials were undertaken and specific treatment with antitoxins was used in patients with toxic clinical cases, having satisfactory results. Convalescent patients were controlled in order to stop the spread of the infection. After approximately 3 years, this disease returned to its regular endemicity.

Key words: Scarlet fever, disease outbreaks, antitoxin.

Palabras clave: Escarlatina, epidemia, antitoxina.

\section{Antecentes históricos}

V on Bergmann en su Tratado de Medicina Interna en el tomo I sobre Enfermedades Infecciosas, versión española del año 1942, decía sobre la distribución geográfica de la escarlatina, "que también en Sudamérica se han descrito epidemias graves, como

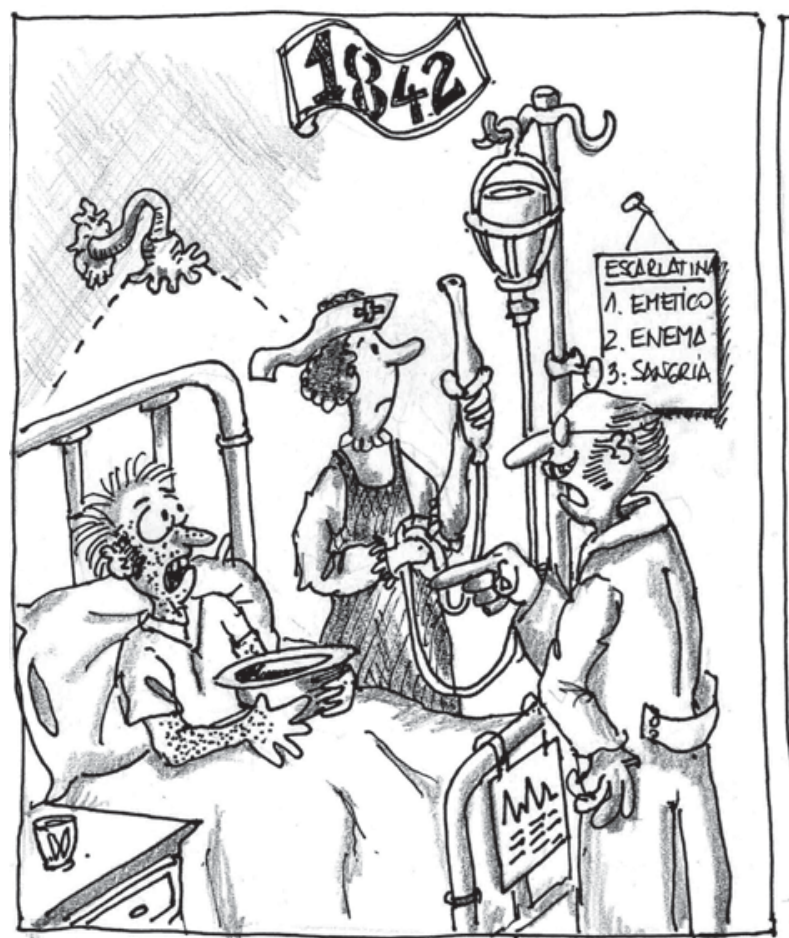

la de 1929, en Chile"1. Esto llevó a interesarme en dicho episodio epidemiológico, recordando que en el año 1827, por primera vez en Chile, se mencionaba la aparición de la escarlatina, según informe del Protomedicato, publicado en los Archivos de la Facultad de Medicina.

Cuatro años después hubo una epidemia que se inició en Valparaíso y pasó a Santiago produciendo en

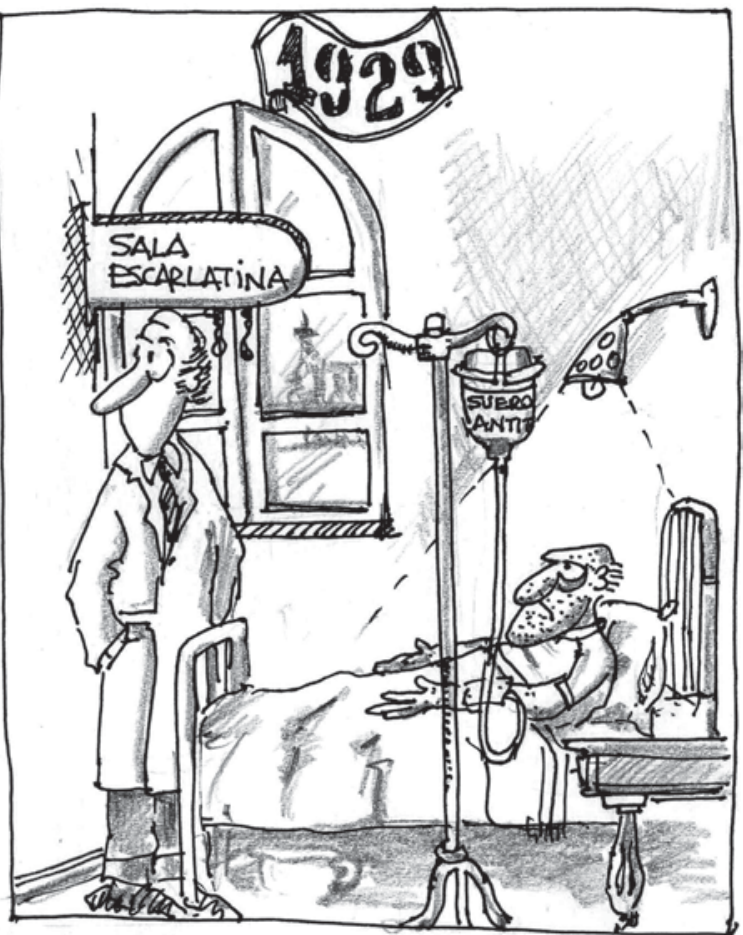


dos años (1831 y 1832), cerca de 7.000 muertos. Según lo anotó el doctor Guillermo Blest, la causa fue "un miasma debido a acumulaciones excrementicias en las quebradas y en otros parajes, por la ausencia de agua y de vientos del sur, así como por un estado particular atmosférico que predisponía a la población a ser afectada, por el miasma".

Otra epidemia acontecida en 1842 llevó al Gobierno a solicitar al doctor Nataniel Miers Cox, en su calidad de Protomédico, que divulgara medidas de prevención y de tratamiento de la escarlatina, que clasificó en simple, anginosa y maligna, manifestando "que era imposible detallar una terapia única justamente por las distintas modalidades clínicas de la enfermedad". En todo caso el doctor Guillermo Blest, más drástico, aconsejaba en todas las formas "sangrías generales $y$ tópicas, las que debían repetirse junto con el baño tibio, vomitivos y purgas frecuentes",2,3.

Durante 1875 y 1876, el doctor Serapio Lois comunicó brotes localizados en Copiapó y Caldera, respectivamente, con escasa mortalidad. A su vez el doctor Arturo Scroggie manifiesta que entre 1889 y 1891, otro brote epidémico de escarlatina, conjuntamente con uno de difteria causó gran número de víctimas "sobre todo entre la gente de la clase acomodada", afirmando que los datos epidemiológicos, así como su mortalidad eran desconocidos y que desde "1891 hasta el año 1929, solo se presentaban casos esporádicos y muy benignos de esta ahora grave enfermedad exantemática",2,3,4

De los datos históricos mencionados, como de las no muy confiables estadísticas de los últimos años del siglo XIX y primeros del siglo XX, se desprendería que el mal ha evolucionado hacia una mayor benignidad, fenómeno que han anotado investigadores de distintas partes del mundo, como que la mortalidad del 15\% a mediados del siglo XIX disminuyó al 2\% en 1900.

Desde que la escarlatina fue individualizada y caracterizada como entidad nosológica, se han mencionado diferentes epidemias que atacan de preferencia los países templados y cuando llegan a regiones tropicales, desaparecen en corto tiempo. En Chile las epidemias han sobrevenido con cierta regularidad y durante los períodos que podrían llamarse de "calma", también se han registrado casos, especialmente en las grandes ciudades. De acuerdo con esto, se puede decir que la escarlatina es una afección endémica, que sufre exacerbaciones periódicas, cuya intensidad llega a constituir, a veces, verdaderos brotes epidémicos ${ }^{5}$.

\section{La epidemia de 1929}

Según Sroggie, los datos de morbilidad en nuestro país eran totalmente inexactos, aun en lo que respecta a enfermedades infectocontagiosas denunciables, de- bido a fallas en la notificación. Tan solo la internación hospitalaria y las estadísticas de mortalidad proporcionarían una idea aproximada sobre la expansión e importancia de la epidemia en estudio. Por lo tanto, para apreciar la grave epidemia de escarlatina, del año 1929, hay que referirse a los datos de los hospitalizados y fallecidos por dicha enfermedad y en ese período.

Durante el decenio 1921-1931, el número de enfermos con escarlatina en los distintos hospitales de la República, hasta el año 1927, fluctuó entre 15 y 65 por año. Desde 1928 comienza a aumentar hasta llegar a 2.551 en el año 1929, para disminuir bruscamente a la tercera parte en el año siguiente y a. la décima en 1931.

Benjamín Viel cita la estadística de mortalidad proporcionada por la Dirección General de Sanidad, no considerando la de morbilidad. En el decenio de 19171927, la mortalidad por escarlatina en todo el país alcanzó a 580 fallecidos. En cambio, tan solo en el año 1929, fallecieron 978 individuos, correspondiendo 537 a la provincia de Santiago (52,5\%), lo que traduciría la gravedad de la epidemia en la capital. El año 1930 hubo 346 fallecidos en todo Chile, con 86 en Santiago (29,7\%), descendiendo la morbilidad de acuerdo a lo mencionado más arriba ${ }^{4,5}$.

Durante el período de 38 años (1898 a 1929), en que sólo se presentaron casos esporádicos y muy benignos de esta entonces temida enfermedad, señala Scroggie algunas consideraciones que sería interesante exponer: "desde luego para la mayoría de los estudiantes de medicina, la escarlatina era conocida por la referencia que de ella se hacía en la llamada clase de patología interna. Los profesores de Clínica Médica y Pediatría no tenían ocasión, por la escasez de estos enfermos, de presentarles a sus alumnos casos con esta patología. Además la falta, de una cátedra especial de enfermedades infecciosas o un hospital para la atención exclusiva de estos pacientes, hace que no tengan los estudiantes y la mayor parte de los médicos jóvenes, oportunidad de conocer y tratar debidamente este importante grupo de enfermedades". Hasta aquí lo escrito por el doctor Arturo Scroggie en 1932, quien se adelantó en algo más de 15 años a la creación de una Cátedra de Enfermedades Infecciosas y de un establecimiento hospitalario para enfermos con estas patologías, a cargo de los profesores Abraham Horwitz y Roque Kraljevic ${ }^{4,6}$.

\section{Medidas para enfrentar la epidemia de escarlatina en 1929}

Dentro de los servicios de Beneficencia y Asistencia Social, la epidemia de escarlatina produjo una situación extraordinaria, ya que aparte de la tarea rela- 
cionada con la profilaxis y medidas preventivas, desarrolladas por la Dirección de Sanidad, le correspondió a la Beneficencia el aislamiento y tratamiento de los enfermos.

En cuanto apareció la escarlatina, con los caracteres que amenazaban transformarla en epidemia, se habilitaron 110 camas en los Hospitales Manuel Arriarán y Roberto del Río. Luego 100 más en el Hospital San Francisco de Borja, destinándose también 120 camas en el Hospital San José. Esta dotación se hizo insuficiente por lo que provocó una reunión de los directores de hospitales, presidida por el Ministro de Bienestar Social don Luis Carvajal, para tratar acerca de las medidas a adoptar, estimándose la "conveniencia de concentrar la atención en determinados hospitales y no difundirla a todos los establecimientos, para evitar el contagio a los demás pacientes".

Se acordó destinar el patio No. 4 del Asilo de Temperancia (en el antiguo Manicomio Nacional), que podría ser aislado perfectamente de las dependencias restantes, trasladándose los "insanos" que actualmente lo habitaban al fundo El Peral (donde se estaba instalando el Open Door y que ahora es la ubicación del Hospital Sótero del Río). Ahí se colocarían 150 camas destinadas a hombres y niños con escarlatina.

Como se habilitaron además 40 camas en el Hospital Roberto del Río y otras 38 en el San Francisco de Borja y contadas las del Asilo de Temperancia, se dispuso en Santiago de 558 camas para los enfermos de escarlatina.

En cuando a las provincias, las Juntas de Beneficencia de las localidades donde se presentó la enfermedad, tomaron las medidas necesarias para el aislamiento de los enfermos mediante sus propios recur$\operatorname{sos}^{7}$

Convendría recordar que en esa época y como sucedió en el Primer Congreso de Cirugía, Medicina Naval y Militar de Chile, en 1929 se definía a la escarlatina como "enfermedad infectocontagiosa por germen desconocido, endémica y que adquiere caracteres de mayor importancia cada cuatro o cinco años". Aunque en el último Congreso de Königsberg (1925) se aceptó como germen productor de la enfermedad al estreptococo hemolítico, aún hay muchos autores que no lo aceptan como tal y que consideran el agente infeccioso todavía desconocido. En 1927 Mandelbaum, cree que el agente específico de la escarlatina es un bacilo difteroide que cuando se asocia al estreptococo hemolítico, aumenta recíprocamente su virulencia y agrega que si el organismo se encuentra inmunizado contra el estreptococo, la enfermedad se presenta bajo la forma de un coriza sanguinolento, de una angina o de otra manifestación difteroidea. En el Congreso señalado se "estimó que la contagiosidad de la escar- latina era mayor por el pus de la otitis, solicitándose que el periodo oficial de aislamiento fuera prolongado a ocho semanas".

Finalmente se concluyó "que según parece desprenderse de las últimas comunicaciones, todos los problemas epidemiológicos relacionados con la escarlatina tienden a solucionarse con el empleo de la vacuna, y sueros preventivos".

\section{Profilaxis de la escarlatina, control de} portadores convalecientes y uso del suero especifico anti escarlatinoso en la epidemia de 1929

El doctor Enrique Onetto, jefe del Departamento de Bacteriología y Diagnóstico del Instituto Bacteriológico de Chile, comunicó el resultado de la vacunación con anatoxina escarlatinosa, efectuada en Santiago, en dos series: una primera constituida por 686 personas que recibieron tres dosis, con una morbilidad de $2,17 \%$, sin mortalidad. Un segundo grupo compuesto por 352 individuos que se vacunaron con dos dosis, siendo la morbilidad de 1,29\%, también sin mortalidad. La morbilidad general de la escarlatina para Santiago, entre enero y mayo de 1929, lapso en que se efectuó la experiencia, se consideró en $18,6 \%$, con una mortalidad de $6,6 \%$.

Los resultados "fueron un poco mejores en cuanto a morbilidad en la serie que recibió dos dosis, lo que Onetto atribuyó al menor número de casos controlados". En general consideró que la vacuna era inocua, y que producía, las mismas reacciones locales y generales que suelen observarse con otras ya conocidas.

Concluye Onetto, "que la vacunación antiescarlatinosa practicada con toxina titulada es eficaz, porque disminuye enormemente la morbilidad y porque la mortalidad llega a cero, debiendo utilizarse como uno de los medios cientificos de profilaxis, en un plan para controlar esta epidemia".

En un grupo de 630 convalecientes examinados, se comprobó desaparición del estreptococo de la faringe, entre 20 y 60 días. Dos casos excepcionales demoraron 70 y 80 días, respectivamente. El microorganismo desapareció en muchos casos antes de los 40 días, lo que permitió concluir que la cuarentena como medida profiláctica es funesta, porque a veces hace perder tiempo inútilmente y otras se da de alta a individuos presumiblemente contagiantes. Según el doctor Onetto, la eficacia del sistema de profilaxis por investigación del estreptococo, la ha palpado en las escuelas donde no se permitió volver a los alumnos sino con certificado en que constaba, no haberse encontrado el estreptococo hemolítico por tres veces seguidas. En todas 
esas escuelas se pudo apreciar que no se presentaron más casos después de la vuelta de los convalecientes aislados y con certificado. En cambio, en aquellos en que se utilizó el procedimiento de la cuarentena, se siguieron presentando.

Onetto piensa "que la investigación del estreptococo hemolitico deberá ser uno de los métodos más recomendables para impedir la difusión del contagio, practicándose sistemáticamente en todos los convalecientes antes de permitir su vuelta a la vida de relación" ".

Durante la epidemia de escarlatina de fines de 1928 y todo el año 1929, se estudió su tratamiento con el suero preparado a partir de cepas de estreptococo hemolítico aisladas de la secreción faríngea de enfermos de escarlatina, apreciándose con la mayor exactitud el beneficio para el enfermo, las condiciones óptimas de su aplicación y mejor aprovechamiento.

El primero que investigó la antitoxina escarlatinosa y su utilización en la curación de la enfermedad fue Moser, quien en Viena el año 1902, preparó a partir de una cepa de estreptococo extraída de un enfermo de escarlatina, suero antiescarlatinoso, empleándolo en 81 enfermos, inyectándoles entre 30 y $80 \mathrm{cc}$, obteniendo un franco retroceso del exantema, descenso de la temperatura y mejoría del estado general. Anterior a Moser, Marmoreck había utilizado suero a partir de cepas de estreptococo hemolítico aislados de otras infecciones no escarlatinosas. Podría mencionarse también a Weissbecker, que en 1897 usó suero de convaleciente. Estos sueros fueron olvidados, siendo necesaria, la aparición de nuevas epidemias para que se les volviera a estudiar. Los fracasos que se produjeron fueron debidos al empleo de preparados con un poder antitóxico bajo, cualidad que no supieron medir.

Así se llegó al año 1924, en que Dochez y Shermann, junto con la aparición de los trabajos de los norteamericanos sobre la etiología estreptocócica de la escarlatina, comunicaron la preparación de un suero con nuevos métodos. Los Dick dieron a conocer un suero curativo con alto poder antitóxico. Con el correr de los años se van sucediendo nuevas experiencias con resultados favorables, destacándose la franca acción sobre la curva febril, con descenso en crisis del alza térmica, a las 24 horas o antes, en la casi totalidad de los casos, hecho que no podía considerarse espontáneo. Al mismo tiempo llama la atención que los síntomas de intoxicación, de postración, debilidad vasomotora, cianosis, sensorio embotado, acompañantes de la escarlatina tóxica, también desaparecían, adoptando el enfermo el aspecto de un convaleciente. El exantema se desvanecía por la acción antitóxica, en la gran mayoría a las 24 horas de su aplicación.

En un período de 10 meses, en los años 1928 y 1929, ingresaron al Servicio de Aislamiento de la Clínica Pediátrica del profesor Scroggie, en el Hospital Roberto del Río, 214 enfermos de escarlatina, siendo tratados con suero antiescarlatinoso específico 59 pacientes graves o con mediana gravedad. A pesar del suero fallecieron $3(5,1 \%$.) De los 155 no tratados, fallecieron 14 (9\%). De este grupo, 32 ingresaron pasada la segunda semana de enfermedad, solamente para curar sus complicaciones. Se comprobó que la eficacia del suero disminuía en razón directa con la tardanza de su utilización. Siempre bastó una sola inyección, con volumen y dosis antitóxica suficiente, cuando se realizó en el transcurso de los primeros tres días de enfermedad.

El profesor Scroggie, gran partidario del suero antitóxico escarlatinoso, aseguraba: "en aquellos casos hipertóxicos, la inyección intravenosa del suero podía ser salvadora".

El doctor Edmundo Cardemil, ejecutor principal de la experiencia terapéutica descrita, creía "que era exagerado el temor que cohibía a muchos clínicos en la aplicación del suero: los accidentes anafilácticos tardios, que según ellos suelen suceder en ocasiones, son más molestos que la misma enfermedad". Sin embargo, Cardemil sólo los había observado en 2\% de los enfermos tratados. Un hecho curioso señalado por el doctor Arturo Scroggie, era que "los niños rubios estarían más expuestos que los morenos a la aparición de reacciones anafilácticas".

En resumen, la indicación del suero se limitaba a los casos de escarlatina tóxica e hipertóxica, dentro de los primeros cinco días de la enfermedad, enfatizándose en la normalización de la temperatura en crisis, desaparición precoz del exantema y de las manifestaciones del compromiso del sistema nervioso, así como la mejoría de las alteraciones vasculares periféricas.

La manera más adecuada de apreciar la calidad del suero y su valor antitóxico, era mediante el fenómeno de la seroextinción del exantema o "blanqueo de la erupción", conocida también como intradermoreacción de Schulz-Charlton ${ }^{10,11,12}$.

En los años siguientes la escarlatina continuó produciéndose, con las conocidas características de una endemia de formas clínicas, por lo general, benignas y con aparición de brotes de diferente gravedad, más o menos cada 4 ó 5 años. Sería importante destacar el de 1937 a 1939, en que se notificaron 5.863 enfermos, correspondiendo el mayor número al año 1939 (2.793), falleciendo 167 pacientes. El último brote que conviene señalar fue el de 1961-1962, con 7.304 enfermos, de los cuales 4.058 sucedieron en 1962, con 38 fallecidos.

En todo caso, la mayoría de las formas clínicas han sido benignas, lo que se ha interpretado como que las cepas del estreptococo $\beta$ hemolítico del grupo A, productoras de la toxina eritrógena $\mathrm{A}$, responsable de los 
cuadros clínicos más graves han ido desapareciendo, con prevalencia de las productoras de toxina $\mathrm{B}$ o $\mathrm{C}$, causantes de los cuadros leves o moderados.

Desde el año 1998 la importancia de la notificación de enfermos con escarlatina, en Chile, ha cedido su lugar a otras enfermedades transmisibles.

Se pensó que desde la introducción de la penicilina las infecciones graves estreptocócicas, habrían llegado a ser casi excepcionales; algunos creyeron que estarían en franca extinción, pero, al igual que en otros países, en el decenio 1985-1995, en diferentes centros asistenciales de Chile, se comprobó un aumento de diversas estreptococcias cutáneas, con la sorprendente aparición de infecciones gravísimas necróticas de tejidos blandos y septicemias similares a las descritas hace varias décadas, algunas de diagnóstico difícil, de evolución fulminante y localizaciones excepcionales.

Sin embargo, manifestaciones de procesos graves escarlatinosos, no se observaron, tanto a nivel local como nacional ${ }^{13,14}$.

\section{Resumen}

La escarlatina es una enfermedad endémica en Chile, de carácter leve a moderado, con morbilidad relativamente baja y exacerbaciones periódicas, cada 4 ó 5 años, de intensidad variable, llegando a constituir, a veces, verdaderos brotes epidémicos. Durante el decenio 1921-1931, el número de enfermos hospitalizados en los distintos establecimientos del país, hasta 1927, osciló entre 15 y 65 por año. A fines de 1928 y durante todo el año 1929, se produjo una epidemia, con casi 3.000 enfermos, falleciendo 978, de los cuales 537 correspondieron a la provincia de Santiago (52,5\%). Las autoridades de Salud Pública (Sanidad, Beneficencia y Asistencia Social), enfrentaron la emergencia con medidas de prevención, profilaxis, aislamiento y tratamiento, habilitándose en la capital 558 camas para la hospitalización de los enfermos. Se emprendieron ex- periencias de vacunación y además de tratamiento para las formas clínicas tóxicas e hipertóxicas con suero antiescarlatinoso específico antitóxico, con resultados satisfactorios. También se realizó control de portadores convalecientes, con el fin de impedir la difusión del contagio. La enfermedad volvió a su endemia habitual al cabo de más o menos tres años.

\section{Referencias}

1.- Von Bergmann G. Tratado de Medicina Interna. Enfermedades Infecciosas. Tomo I. p. 359. Ed. Labor S.A. Barcelona. España. 1942.

2.- Laval R E. Notas históricas sobre escarlatina. Rev Chil Infect 1984; 2: 135-6.

3.- Olea N A. Historia de las Enfermedades Infecciosas en Chile. El Vigía 2000; 3: 56.

4.- Scroggie V A. La escarlatina. Arch Hosp de Niños Roberto del Río 1932; 3: 133-45.

5.- Viel V B. Epidemiología de la escarlatina en Chile. Rev Chil Ped 1939; 9: 699-714; 781-810; 844-55.

6.- Kraljeviæ O R. Recuerdos de un viejo infectólogo. Contribución a la Historia de la Infectología en Chile. (1937-1970). Pfizer. Santiago de Chile. 1998.

7.- Bravo G A. La epidemia de escarlatina. Medidas para hacerle frente. Bol de Benef 1929; 1: 19.

8.- Marín G R. Primer Congreso de Cirugía, Medicina Naval y Militar de Chile. La Clínica 1929; 5: 1364-76.

9.- Onetto A S. Profilaxis de la escarlatina y control de portadores convalecientes en la pasada epidemia. Rev Inst Bact Chile 1929,1: 80-6.

10.- Cardemil M E. La escarlatina y su tratamiento por medio del suero específico antiescarlatinoso. Arch Hosp de Niños Roberto del Río 1930-31; 2: 155-68.

11.- Scroggie V A. La escarlatina (continuación). Arch Hosp de Niños Roberto del Río 1933; 4: 57-66.

12.- Ortega I G. La escarlatina. Trabajos y publicaciones de las Clínicas del Hospital Roberto del Río. Arch Hosp de Niños Roberto del Río 1930; 1: 49-54.

13.- Anuario de Enfermedades de Notificación Obligatoria. 1932-1997. Dirección de Sanidad, Servicio Nacional de Salud, Ministerio de Salud, Chile.

14.- Laval R E. La infección por el estreptococo beta hemolítico del grupo A: ¿vuelta al pasado? Rev Chile Infect 1994; 11: 73-81. 